\title{
Marfan syndrome in a large family: response of family members to a screening programme
}

\author{
A B Bridges, M Faed, M Boxer, J R Gray, C Bundy, A Murray
}

\begin{abstract}
Reaction to medical, social, and genetic implications of Marfan syndrome was evaluated by means of two questionnaires, the first after various tests before discussion of the diagnosis, the second after full discussion of the patient's diagnosis.

Thirty-seven members of a family known to be at risk for Marfan syndrome attended for both questionnaires. All patients claimed to be satisfied with the way they were informed of the results of screening; $41 \%$ of patients were more worried about their health and $48 \%$ were more worried about the future after diagnosis.

Apart from $50 \%$ of the smokers reducing or stopping their intake of cigarettes there were only very minor changes in lifestyle over the first month despite the increased level of expressed anxiety. If a definitive screening test was available, $96 \%$ of patients claimed they would have chosen it, $45 \%$ felt it would have an influence on their future plans, and $78 \%$ would choose to use a method of prenatal diagnosis for Marfan syndrome if it were available.
\end{abstract}

Marfan syndrome is an autosomal dominant heritable disorder of connective tissue with variable expressivity. The major clinical manifestions involve the ocular, skeletal, and cardiovascular systems. Previous studies that described the natural history of this disorder have reported that the mean age of death is in the early thirties and $87 \%$ of these deaths occurred as a result of cardiac complications. However, the survival of Marfan syndrome patients has improved significantly with regular echocardiographic surveillance, beta blockade, and prophylactic repair of dilated aortic roots. ${ }^{2}$

A screening programme was initiated in a large family after the presentation and diagnosis of a subject with Marfan syndrome. ${ }^{3}$ From the medical point of view, there are clear benefits for affected subjects associated with early diagnosis, subsequent follow up, and treatment if required. However, although screening may be clinically beneficial to the patient(s) for whom a definite diagnosis can be provided, there may be a number of associated drawbacks with respect to possible employment, financial, psychological, and social problems for both the affected subject and his family. In addition, for some patients the diagnosis after screening may remain uncertain.
From the patient's viewpoint, the benefits associated with screening and a diagnosis of Marfan syndrome may therefore not be so clear cut.

None of the family members in this screening programme was symptomatic nor had sought medical attention, with the exception of the proband who had presented as an emergency and one of his brothers and a son. They had previously been screened for Marfan syndrome but were not attending regular follow up clinics.

This paper reports on the perceived advantages and disadvantages of screening from the family's viewpoint. In order to ascertain the attitudes towards the screening programme, members of the family at risk were requested to complete two questionnaires, the first after they had been screened but before they had been informed of the result and the second after they had been informed of the result. The aims of the questionnaire were to assess the family's understanding of the inheritance of Marfan syndrome, to elicit their attitudes towards the screening programme, to gauge their satisfaction with the service, and, finally, to note any lifestyle changes induced by participation in the screening programme.

The views expressed by members of this large family should be of interest to those who provide care and counselling for Marfan syndrome families.

\section{Patients and methods}

A 39 year old man was admitted with a dissecting aortic aneurysm and examination also showed the skeletal stigmata of Marfan syndrome based on the criteria described by Pyeritz and McKusick. ${ }^{4} \mathrm{He}$ was transferred urgently to a cardiothoracic unit where an emergency repair of the aneurysm and replacement of the aortic valve were performed. $\mathrm{He}$ was subsequently discharged but was readmitted four years later with dehiscence of the aortic graft. This was repaired and he was later transferred back to his referring hospital for postoperative convalescence. A full family pedigree was then obtained.

Thirty-nine members of his extended family ranging from 1 to 77 years of age in four generations were ultimately identified as being at risk of Marfan syndrome. ${ }^{3}$ These were all either first or second degree relatives of an affected subject. They were invited to attend as outpatients to be screened for the clinical signs of Marfan syndrome. ${ }^{45}$ Details of the screening programme visits are shown in table 1 . Thirtyeight members of the family attended. The 
nature of the disorder, its potential complications, possible treatments, and the purpose of screening were discussed with each patient or the parents of young children who attended. The physical and echocardiographic examinations necessary for a diagnosis were described and it was explained to the patients that a diagnosis would be available after the ophthalmological appointment. It was made clear that all results would be explained to them and counselling would be available after the screening procedure. Following consent, each patient was examined for the cardiovascular and skeletal features of Marfan syndrome and an echocardiogram was performed. Each patient was then referred for an ophthalmological opinion, the time from referral until the outpatient appointment being approximately three months. The patients were then seen again by the same physician who had performed the skeletal and cardiac examinations. Their diagnosis was discussed with reference to the cardiovascular, skeletal, ophthalmological, and echocardiographic examinations. Each patient had the diagnosis fully explained in lengthy, confidential discussions. Patients who were diagnosed as not having Marfan syndrome were relatively simple to counsel with discussion of lack of risk to themselves and their offspring. Patients newly diagnosed as having Marfan syndrome were seen in serial appointments during which the problems relating to offspring, occupation, and financial matters, including insurance and mortgages, were discussed. Each patient with Marfan syndrome was given a Marfan Association booklet and encouraged to bring any problems back to subsequent sessions. Patients were made aware of support groups, such as the Marfan Association and its value as a source of information relating both to the syndrome and also sources of financial help such as sympathetic insurance brokers. The counselling of patients in the uncertain group was difficult. Our practice was to follow guidelines suggesting patients over 20 years of age with a normal echocardiogram had an excellent prognosis. ${ }^{6}$ The patients were advised as such and no reference was made in letters or notes to Marfan syndrome, the condition being described as 'Marfanoid'. The screening programme showed 12 members of

Table 1 Timetable of screening programme visits.



the family unequivocally to have Marfan syndrome. This group included the proband, one of his sons, a brother, eight newly diagnosed adults, and one newly diagnosed child. Furthermore, examination of the death certificates of two of the proband's sibs gave information which indicated that these subjects had also had Marfan syndrome. There were four members of the family in whom a definite diagnosis could not be made on clinical grounds.

Within four weeks of their first visit, the proband and the 26 relatives who had been screened and were aged 16 years of age or over attended as outpatients to complete the first questionnaire assessing their attitudes to the screening programme. At the time the first questionnaire was completed, only four members of the family knew their own diagnosis; they were the proband, his son and brother who had previously been screened but were not attending regular outpatient clinics, and an asymptomatic sister. The latter had an aortic aneurysm detected at echocardiography (diameter $5.8 \mathrm{~cm}$ ) and required further investigations before her ophthalmology appointment.

Each relative completed the questionnaire alone and in his/her own time. The questionnaire collected demographic information, details of person's knowledge of Marfan syndrome, and the attitudes of the family to future possible diagnostic tests. Patients reported the anxiety induced by the programme and the perceived advantages and disadvantages of screening.

The questionnaire contained two types of question. The majority of questions required the patient to choose an option from a selection of possible answers. Three questions were of an open format and required the patients to formulate their own answers. These questions related to the advantages and disadvantages of screening and to suggestions for improvements to the screening programme.

The second questionnaire was completed four weeks after the patients had been informed of their diagnoses. It contained two open format questions to ascertain the advantages and disadvantages of screening for Marfan syndrome from the patients' point of view and a question which asked the respondent to select an answer as to whether or not it had been in his/her own interest to have been screened and informed about Marfan syndrome. These particular questions were presented in exactly the same format in both questionnaires.

\section{Results}

\section{DEMOGRAPHIC DETAILS}

The 27 members of this family who completed the first questionnaire ranged in age from 16 to 77 years of age. Fourteen of these patients were men. Six of the group were in social class II and 21 in social class III as assessed by the Registrar General's classification. The 21 respondents to the second questionnaire ranged in age from 16 to 77 . There were 12 males and five of this group were in social class II. 
QUESTIONNAIRE 1 RESPONSES

Knowledge about Marfan syndrome

Ten patients stated that they knew before attendance at the screening clinic that this disorder could be inherited. Twenty subjects first heard about Marfan syndrome from relatives, six from the screening clinic, and four from a doctor (some relatives answered two options). Within a period of one month of being informed of the nature of Marfan syndrome, its mode of inheritance, and their own particular risk, 26 relatives knew this was an inherited disorder, 24 thought they were at risk of having Marfan syndrome, and 16 assessed their own a priori risk of having the disorder correctly.

\section{Satisfaction with the service}

All 27 stated they were satisfied with the way in which they had been informed about Marfan syndrome. In response to a question asking for suggestions to improve the service, one relative suggested that more information be given to patients and another stated that the family should have been informed earlier.

\section{Attitudes to potential diagnostic tests}

The 23 relatives who did not know their own diagnosis were asked whether they would have wished to undergo diagnostic testing for Marfan syndrome had such an assessment been available. Of these, 22 replied affirmatively and all 23 indicated that they would wish to know the result of the test themselves. Ten patients indicated that the diagnostic test result would have influenced their own plans for the future, while 11 also stated it would influence their plans to have or have had children. If a method of prenatal diagnosis for Marfan syndrome were available, 18 stated they would wish to make use of it in planning a family.

\section{Anxiety induced by the screening}

Of the 27 relatives, 11 were more worried about their health after screening and 13 were more worried about their future than before screening. Indirect methods used to assess anxiety after screening asked questions about lifestyle changes. Within four weeks of screening, 24 recorded no change in level of exercise and 23 no dietary changes. Of the 14 subjects who smoked cigarettes before screening, seven stated that they had either reduced the number of cigarettes smoked or stopped altogether. Twenty-two reported there was no change in alcohol consumption and five that it had decreased.

\section{Responses of the patients who knew their} diagnosis at the time of completing the first questionnaire

The responses of these four patients were examined separately. All stated that they would make use of a prenatal diagnostic test in planning a family if it were available. The advantages associated with early diagnosis and treatment were identified as the main benefit of screening by all the patients. One subject identified anxiety as a potential disadvantage to screening. All of this group indicated that they would attend regular outpatient follow up. Three felt it was "definitely in their own interest" to have been informed about Marfan syndrome, the other stated that it was "probably in his/her own interest".

After screening, three recorded no change in the level of exercise taken, worry about health, diet, or relationship with family. Two were non-smokers and the other two stopped smoking. Two stated that they were more worried about the future than before screening and one less.

\section{QUESTIONNAIRE}

The responses to this questionnaire are detailed in table 2. This questionnaire was completed by patients four weeks after they had been informed of their diagnosis and 21 out of 27 members of the family completed it. The six patients who did not complete the second questionnaire were all diagnosed as definitely not having Marfan syndrome. The responses of the four patients who knew their own diagnoses before the initiation of the screening programme have been excluded from the results presented in table 2 .

\section{Discussion}

Before the screening programme, 10 members of this family knew of the existence of Marfan syndrome and its genetic inheritance. However, only three of these subjects had previously been screened, the proband, one of his sons, and a brother. The proband had another son who had not been screened. The questionnaire did not elucidate the reasons for this.

Within one month of the family having been informed verbally about Marfan syndrome, an assessment of their knowledge indicated that most of the patients had understood the genetic basis of the disease and were able to state accurately their own a priori risk.

At the moment no routine laboratory diagnostic test exists for Marfan syndrome. However, the location of the gene defect causing this disorder has recently been reported ${ }^{78}$ and the gene for fibrillin, which has been implicated in Marfan syndrome, ${ }^{9}$ has been mapped to this location. ${ }^{10}$ The patients' response to the questionnaire suggests that if a diagnostic test were to become available, the uptake among this family would be high. Almost half stated that the results would be taken into consideration when making major decisions about their future.

In clinical practice, it would be especially valuable to have a diagnostic laboratory test for the family members in whom Marfan syndrome cannot definitely be confirmed or excluded on clinical criteria. The screening programme detected four adults in this family, three postmenopausal women and a 21 year old male, in whom no clear clinical diagnosis was possible. These patients were informed that 
Table 2 Details of the responses to questions concerning the advantages, disadvantages, and whether the patients thought it was in their own interest to have been screened for and informed about Marfan syndrome. The patients completing both questionnaires were divided into three groups by their final diagnosis, definite Marfan syndrome $(n=4)$, definitely not Marfan syndrome $(n=9)$, and possible Marfan syndrome $(n=4)$. None of these patients knew their diagnosis while completing the first questionnaire and they all knew their diagnoses when they completed the second questionnaire.

\begin{tabular}{|c|c|c|c|c|c|c|}
\hline & \multicolumn{2}{|c|}{$\begin{array}{l}\text { Definite MS } \\
\quad(n=4)\end{array}$} & \multicolumn{2}{|c|}{$\begin{array}{l}\text { Definite not } M S \\
(n=9)\end{array}$} & \multicolumn{2}{|c|}{$\begin{array}{l}\text { Possible MS } \\
(n=4)\end{array}$} \\
\hline & Pre & Post & Pre & Post & Pre & Post \\
\hline $\begin{array}{l}\text { Advantages } \\
\text { Early treatment } \\
\text { Able to change lifestyle } \\
\text { Able to support relatives } \\
\text { Knowledge that they are unaffected } \\
\text { None } \\
\text { No answer given }\end{array}$ & $\begin{array}{l}\frac{1}{-} \\
\frac{-}{3}\end{array}$ & $\begin{array}{l}\frac{1}{2} \\
\frac{1}{3}\end{array}$ & $\begin{array}{l}\frac{8}{-} \\
\frac{1}{-}\end{array}$ & $\begin{array}{l}5 \\
2 \\
2 \\
4 \\
- \\
-\end{array}$ & $\begin{array}{l}\frac{2}{-} \\
\frac{-}{2}\end{array}$ & $\begin{array}{l}\frac{2}{-} \\
\frac{2}{2}\end{array}$ \\
\hline $\begin{array}{l}\text { Disadvantages } \\
\text { Anxiety } \\
\text { Employment problems } \\
\text { Restriction of lifestyle } \\
\text { None } \\
\text { No answer given }\end{array}$ & $\frac{2}{\frac{1}{1}}$ & $\frac{1}{\frac{1}{1}}$ & $\begin{array}{l}1 \\
2 \\
1 \\
3 \\
2\end{array}$ & $\begin{array}{l}1 \\
2 \\
1 \\
3 \\
2\end{array}$ & $\begin{array}{l}- \\
\frac{-}{1} \\
3\end{array}$ & $\begin{array}{l}\overline{-} \\
\overline{1} \\
2 \\
1\end{array}$ \\
\hline $\begin{array}{l}\text { Has it been in your interest to be screer } \\
\text { informed of your risks of having Marf } \\
\text { syndrome? }\end{array}$ & & & & & & \\
\hline $\begin{array}{l}\text { Definitely yes } \\
\text { Probably yes } \\
\text { Not sure } \\
\text { Probably no } \\
\text { Definitely no }\end{array}$ & $\begin{array}{l}3 \\
1 \\
- \\
-\end{array}$ & $\begin{array}{l}\frac{4}{-} \\
- \\
-\end{array}$ & $\begin{array}{l}8 \\
\frac{1}{-} \\
-\end{array}$ & $\frac{8}{1}$ & $\begin{array}{l}3 \\
1 \\
- \\
-\end{array}$ & $\begin{array}{l}2 \\
2 \\
- \\
-\end{array}$ \\
\hline
\end{tabular}

Pre $=$ before knowledge of diagnosis. Post $=$ after knowledge of diagnosis.

Marfan syndrome could not definitely be excluded, therefore regular outpatient follow up with echocardiograms was recommended. They were also informed that in view of their normal echocardiograms their long term prognosis was good ${ }^{6}$. For the majority of relatives the screening programme had clear benefits. A positive diagnosis allowed initiation of echocardiographic surveillance and early treatment, while a negative diagnosis removed the subjects' doubt and fears that they may be affected. Many relatives also expressed the sentiment that they were pleased that at last something was being done for this family in which at least three members with Marfan syndrome had died suddenly. However, for the group with an uncertain diagnosis the value of the screening programme is more difficult to establish. These patients found it confusing to have the possibility raised that they might have a serious genetic disorder, to consent to and undergo screening for it, and then be informed that an unequivocal clinical diagnosis was not possible. It is therefore important to recognise that while screening programmes may benefit some subjects, they may also create problems for others.

The overall cost-benefit ratio of the screening programme described by the family in questionnaire 1 indicated that all the patients felt it was in their own interest to know of the possible risk of having Marfan syndrome and to be screened for it. However, one patient was not sure of the benefits when the follow up questionnaire was completed. The advantages described by most of the family relate to early diagnosis and treatment. The disadvantages described relate mainly to anxiety about the screening programme and the issue of employment. The responses relating to these issues were similar in both questionnaires. However, some patients described advantages such as "knowing I am not affected" and "being able to support others in the family" in questionnaire 2 which were not recorded in questionnaire 1. Employment problems arose after the screening programme and these were alluded to by the responses in questionnaire 2 . One asymptomatic member of the family who had aortic root dilatation lost his labouring job and another who ran a small gardening business had to alter his work practices as they were advised not to lift heavy weights. However, irrespective of a priori risk and eventual diagnosis, there were no differences in the attitudes of this family to screening.

It is important to be aware of the anxiety which may be induced by a screening programme since this is known to impair the ability of patients to recall advice and information. ${ }^{11}$ However, one month after the screening procedure, the ability of relatives to recall information and advice given at the clinic seemed satisfactory. Although, an increased number of relatives were more worried after than before screening, no major alterations in lifestyles in the first month following screening were recorded. Whether the raised levels of anxiety were considered sufficient to warrant treatment remains uncertain and future research in this field should consider this point. In our screening programme counselling was given regarding the medical aspects of Marfan syndrome, but no attempt was made at this stage to reduce anxiety other than to provide accurate information about Marfan syndrome. However, on the basis of our results it may be advisable for future screening programmes to include some form of anxiety reduction to allow the patients to 'hear' the information being given. This could amount to written information or a video about the purpose, methods, and implications of screening. In addition open access to a specific doctor specialising in genetic disorders would allow patients to feel that they were more in control 
of consultations and able to deal with their concerns as they arose. A further possible method of reducing the anxiety in these patients would be to minimise the waiting times required for the diagnostic examinations to be performed.

Overall, the family appeared satisfied with the screening programme. This may be in part because of the circumstances surrounding this family. These included three deaths from Marfan syndrome, the proband having two major lifesaving operations, the screening programme diagnosing nine new cases, and the subsequent potentially lifesaving prophylactic operation on one of these patients. Other Marfan syndrome families with different incidence of affected members, mortality, or serious complications may have a different view of screening programmes.

One question which often arises after the diagnosis of a serious genetic disorder is whether or not the members of the family at risk should be screened. Any decision is likely to be based on many considerations including the proband's wishes, the nature of the disorder, whether an accurate diagnostic test is available, and if the disorder or its complications are treatable. Marfan syndrome has a high mortality from cardiac complications in adults and children. In a series of 25 children with Marfan syndrome with a mean follow up of five years from the time of diagnosis, the mortality rate was $22 \% .{ }^{12}$ Our screening programme was also attended by 11 children under 16 years of age with their parents. The diagnosis of Marfan syndrome in childhood has important therapeutic implications which may alter the course of the disorder.

Screening at risk family members of a Marfan syndrome patient may allow early initiation of treatment which has been shown to improve survival significantly. ${ }^{124}$ Despite the medical benefits associated with early diagnosis there may be drawbacks for the patient and the doctor should be aware that psychological, financial, or employment difficulties may arise as a consequence of screening.

It is often difficult to know how far into the wider family screening should be extended. The complications of Marfan syndrome are treatable and thus there are clear medical reasons to screen relatives of patients. The results in this paper suggest that almost all members of a large family were very positive towards the screening programme and we see this as further weight to the argument for screening all first and second degree relatives.

We thank the Marfan Association for their assistance.

1 Marsalese DL, Moodie DS, Vacante M, et al. Marfan's syndrome: natural history and long term follow up of cardiovascular involvement. $f \mathrm{Am}$ Coll Cardiol 1989;14:422-8

2 Child JS, Perloff JK, Kaplan S. The heart of the matter: cardiovascular involvement in Marfan's syndrome. $f$ Am Coll Cardiol 1989;14:429-31.

3 Bridges AB, Faed M, Boxer M, Haining WM, Pringle TH McNeill GP. Marfan syndrome affecting 4 generations of a family without ocular involvement. Postgrad Med $\mathcal{F}$

4 Pyeritz RE, McKusick VA. The Marfan syndrome: diagnosis and management. $N$ Engl $\mathcal{F}$ Med 1979;300:772-7. gnosis and management. $N$ Engl f Med 1979;300:772-7.
Super M. Diagnosing Marfan syndrome. BMF 5 Super M. Diagnosing Marfan syndrome. $B M \mathcal{F}$

6 Child AH. Diagnosing Marfan's syndrome. BMF 1988;296:1673-4

7 Kainulainen K, Pulkkinen L, Savolainen A, Kaitila I, Peltonen L. Location on chromosome 15 of the gene defect causing Marfan syndrome. $N$ Engl $f \mathrm{Med}$ 1990;323:935-9.

8 Tsipouras P, Sarfarazi M, Devi A, Weiffenbach B, Boxer $M$. Marfan syndrome is closely linked to a marker on chromosome 15q1.5-q2.1. Proc Natl Acad Sci USA 1991;88:4486-88

9 Hollister DW, Godfrey M, Sakai LY, Pyeritz RE. Immunohistologic abnormalities of the microfibrillar-fiber system in the Marfan syndrome. $N$ Engl $f \mathrm{Med}$ 1990;323:152-9.

10 Lee B, Godfrey M, Vitale E, et al. Linkage of Marfan syndrome and a phenotypically related disorder to two different fibrillin genes. Nature 1991;353:330-4.

11 Marteau TM. Reducing the psychological costs. BMF 1990;301:26-8.

12 Geva T, Hegash J, Frand $M$. The clinical course and echocardiographic features of Marfan's syndrome in childhood. Am $\mathcal{F}$ Dis Child 1987;141:1179-82. 